\title{
Presentation, Complication, Management, and Drug Adherence of Patients with Thyrotoxicosis in Developing Countries
}

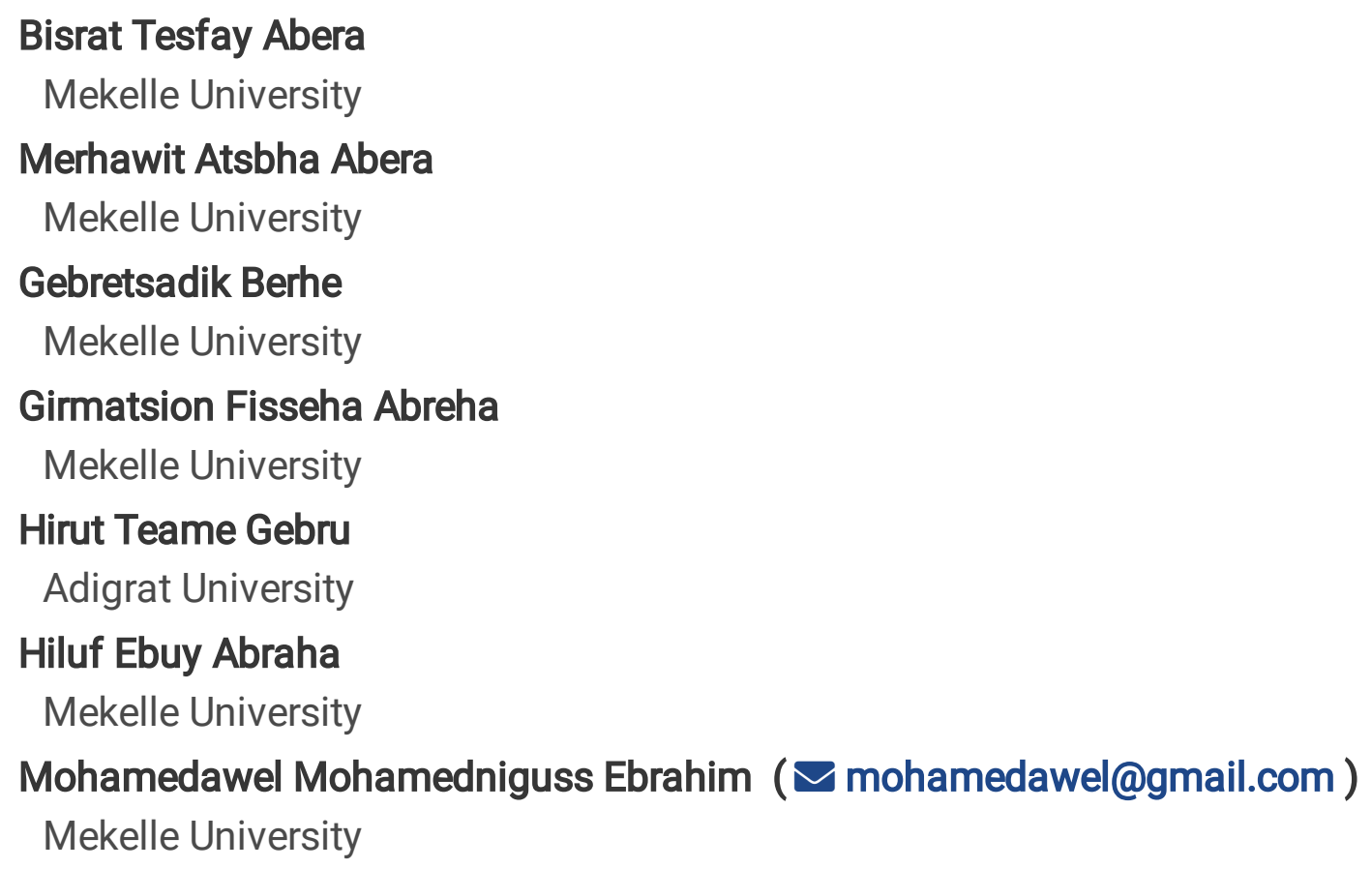

Bisrat Tesfay Abera

Mekelle University

Merhawit Atsbha Abera

Mekelle University

Gebretsadik Berhe

Mekelle University

Girmatsion Fisseha Abreha

Mekelle University

Hirut Teame Gebru

Adigrat University

Hiluf Ebuy Abraha

Mekelle University

Mohamedawel Mohamedniguss Ebrahim ( $\nabla$ mohamedawel@gmail.com )

Mekelle University

\section{Research Article}

Keywords: $\beta$-Blocker, Adherence, Dilated Cardiomyopathy, Thyrotoxicosis, Propylthiouracil, Ethiopia

Posted Date: April 29th, 2021

DOl: https://doi.org/10.21203/rs.3.rs-397103/v1

License: (9) (i) This work is licensed under a Creative Commons Attribution 4.0 International License. Read Full License 


\section{Abstract}

Background: Thyrotoxicosis is the state of thyroid hormone excess with a spectrum of disease ranging from mild to life-threatening complications like thyrotoxic cardiomyopathy and thyroid storm. This study aims to explore the pattern, clinical manifestation, complication, management, and drug adherence of patients with thyrotoxicosis in a tertiary hospital of Northern Ethiopia.

Methods: An institution-based cross-sectional study was conducted in Ayder Comprehensive Specialized Hospital from 2017 to 2018. Data from a 200 thyrotoxicosis cases was collected using a structured questionnaire that includes Morisky Medication Adherence Scale questions. After describing variables, a logistic regression was conducted to identify independent predictors of dilated cardiomyopathy and drug adherence. Statistical significance was declared at $p<0.05$.

Results: Mean age at presentation of thyrotoxicosis was 45 years and females accounted for $89 \%$ of the cases. As well, the most common symptom and sign were palpitation and goiter respectively. Thyroid storm occurred in $6 \%$ of the cases. Out of 89 patients subjected for echocardiography, $35(39.3 \%)$ of them had dilated cardiomyopathy. And, the odds of dilated cardiomyopathy was higher in patients who had atrial fibrillation (AOR=15.95, 95\% Cl:5.89-38.16, $\mathrm{p}=0.001)$ and tachycardia (AOR=2.73, 95\% Cl:1.04-7.15, $p=0.040$ ). All patients took propylthiouracil and $13.0 \%$ of them experienced its side effects. Concerning $\beta$ blockers, propranolol was the most commonly (78.5\% of the cases) used drug followed by atenolol (15.0\%). Six patients underwent surgery. Lastly, drug non-adherence rate was $26 \%$ and it was significantly associated with illiteracy, side effect, lack of information and infrequent TSH follow-up.

Conclusions: In developing countries like Ethiopia, patients with thyrotoxicosis have no access to methimazole which is the first-line anti-thyroid drug. In addition, they greatly suffer from dilated cardiomyopathy due to late presentation and side effects of propylthiouracil. Therefore, we recommend that patients should get adequate health information about thyrotoxicosis and anti-thyroid drugs including their side effects. Additionally, hospitals and other concerned bodies should also avail TSH test and methimazole at an affordable cost.

\section{Background}

Thyroid disorders are common endocrine disorders which affect body metabolism (1). One of these disorders is thyrotoxicosis which is the state of thyroid hormone excess (2). The commonly mentioned causes of thyrotoxicosis are grave's disease, toxic multinodular goiter, and toxic adenoma. In iodinereplete communities, the prevalence of thyrotoxicosis in women is thought to be between 0.5 and $2 \%$, and 10 times more common than men (3). But its prevalence among patients with thyroid disorders may reach up to $62 \%(1)$.

The usual manifestations of thyrotoxicosis are hyperactivity, irritability, dysphoria, heat intolerance, sweating, palpitation, fatigue, weight loss with increased appetite, diarrhea, polyuria, oligomenorrhea and loss of libido(2,4). In addition, patients with thyrotoxicosis could complicate with different disorders one 
of which is thyrocardiac disease that can happen in $27 \%$ of the cases (5). Some of the cardiac complications of thyrotoxicosis are atrial fibrillation, non-specific ST-T changes, ventricular hypertrophy, and dilated cardiomyopathy(5-8). Furthermore, $1 \%$ of patients with thyrotoxicosis could present with a life threatening complication called thyroid storm which has a mortality risk of $10-20 \%(9,10)$.

The thionamide drugs (propylthiouracil (PTU), methimazole, and Carbimazole), beta blockers, radioactive iodine, and surgery are the treatment modalities used in the management of thyrotoxicosis and thyroid storm each with different success rates. Patients with thyroid storm will need further treatment with glucocorticoids and lugol's solution (1,11-16).

In sub-Saharan Africa (SSA), specifically Northern Ethiopia, scientific evidence about thyrotoxicosis is limited. Therefore, this study aims to explore the pattern, clinical manifestation, complication, management and drug adherence of patients with thyrotoxicosis in Ayder Comprehensive Specialized Hospital which is the only tertiary hospital in Tigrai, Northern Ethiopia.

\section{Methods}

\section{Study Area and Period}

An institution-based cross-sectional study was conducted in Ayder Comprehensive Specialized Hospital (ACSH) which is located in Mekelle, Tigray regional state, Northern Ethiopia. It gives both outpatient and inpatient services. One of the outpatient service units is endocrine referral clinic that started giving services since January 2017. It gives monthly follow-up to patients with endocrine disorders at a specialty clinic with 01 internist in charge. This study was carried out from December, 2017 to June 30, 2018.

\section{Source and Study Population}

\section{Source Population}

All patients with thyrotoxicosis who had follow-up at the endocrinology clinic at ACSH.

\section{Study Population}

All patients with thyrotoxicosis who had follow-up at the Endocrinology Clinic at ACSH during the study period.

\section{Eligibility Criteria}

Patients above 18 years of age with thyrotoxicosis who had follow-up at the endocrine referral clinic of ACSH were involved in this study.

\section{Samples}


All thyrotoxicosis patients who visited the endocrinology clinic during the study period were included.

\section{Variables}

\section{Dependent Variables}

The dependent variables were dilated cardiomyopathy, and drug adherence. Medication adherence was assessed using an 8-item Morisky Medication Adherence Scale (MMAS-8). The MMAS-8 scores can range from 0 to 8 points. A patient was considered adherent to antithyroid medication(s) if the MMAS- 8 score was zero or one and non-adherent if the score was above one.

\section{Independent variables}

Socio-demographic factors (age, gender, occupation, income, educational status, residence), clinical features (symptoms and signs), comorbidity, level of T4, and frequency of TSH follow-up were considered as independent variables.

\section{Data Collection}

Data were collected by two trained internal medicine residents through interviewer administered structured questionnaire. The questionnaire was designed to collect data related to sociodemographic characteristics, clinical presentation, investigation, management modality, and drug adherence.

\section{Data Analysis}

Data entry and analysis were performed using Statistical Package for Social Science (SPSS) version 23. Descriptive and summary statistics were used to describe the data in relation to relevant variables. Odds ratio with its $95 \%$ confidence interval and $p$-value were calculated by running logistic regression to identify the presence and strength of association. Variables with a p-value less than 0.05 during bivariate analysis were selected for multivariable analysis. Statistical significance was declared at $p$-value $<0.05$.

\section{Ethical Consideration}

Ethical clearance was obtained from Ethical Review Committee of Mekelle University, College of health sciences with ethical clearance number ERC: 1408/2018. After explaining the purpose of the study, informed consent was obtained from the participants. Confidentiality of information was also assured by removing medical record numbers and replacing them with codes.

\section{Results}

A total of 200 patients with thyrotoxicosis who fulfilled the inclusion criteria were included in this study. 
Socio-demographic characteristics and disease category

There were 178 (89\%) females with male to female ratio of 1:8.1. Age of patients ranged from 18-77 years. The mean age at presentation was $45.2 \pm 14.3$ year. More than half (58.5\%) of patients were unable to read and write. Toxic multinodular goiter accounted for $51.5 \%$ of the cases (Table 1).

Table 1: socio-demographic characteristics of patients with thyrotoxicosis at the endocrine clinic, ACSH from December, 2017 to June, 2018.

\begin{tabular}{ccl}
\hline Variables & Frequency $(\mathbf{N = 2 0 0 )}$ & Percentage (95\% CI) \\
\hline Sex & 178 & $\mathbf{8 9 . 0}(83.8-93.0)$ \\
Female & 22 & $11.0(7.0-16.2)$ \\
Male & & \\
Age (in years) & 83 & $\mathbf{4 1 . 5}(34.6-48.7)$ \\
18-40 & 80 & $\mathbf{4 0 . 0}(33.1-47.1)$ \\
>1-60 & 37 & $\mathbf{1 8 . 5}(13.4-24.6)$ \\
Residence & 100 & \\
Urban & 100 & $\mathbf{5 0}(42.9-57.1)$ \\
Rural & & \\
Educational status & 30 & $\mathbf{1 5 . 0}(10.4-20.7)$ \\
Higher education & 25 & $\mathbf{1 2 . 5}(8.3-17.9)$ \\
Secondary school & 18 & $\mathbf{9 . 0}(5.4-13.9)$ \\
Primary school & 10 & $\mathbf{5 . 0}(2.4-9.0)$ \\
Able to read and write & 117 & $\mathbf{5 8 . 5}(51.3-65.4)$ \\
Unable to read and write & & \\
Income (in ETB) & 106 & $53.0(45.8-60.1)$ \\
<1000 & 46 & $\mathbf{2 3 . 0}(17.4-29.5)$ \\
1000-3000 & 48 & $\mathbf{2 4 . 0}(18.3-30.5)$ \\
>3000 & & \\
Tiagnosis & 102 & $\mathbf{5 1 . 5}(44.3-58.6)$ \\
Toxic multinodular goiter & 75 & $\mathbf{3 7 . 5}(30.8-44.6)$ \\
Gric nodular goiter & 18 & $\mathbf{9 . 0}(5.4-13.9)$ \\
Other & 5 & $\mathbf{2 . 5}(0.8-5.7)$ \\
\hline
\end{tabular}

\section{Fine Needle Aspiration Cytology}

A total of 129 (65\%) patients were subjected to fine needle aspiration cytology (FNAC). The most common FNAC finding was colloid Goiter 104 (80.62\%) followed by atypia of undetermined significance (AUS) 8 (6.20\%) and Grave's disease 3 (2.33\%) (Table 2). 
Table 2: FNAC results of patients with thyrotoxicosis at the endocrine clinic, ACSH from December, 2017 to June, 2018, N=129.

\begin{tabular}{lll}
\hline Cytology & Frequency & Percentage (95\% CI) \\
\hline Colloid goiter & 104 & $80.6(72.7-87.0)$ \\
Grave's disease & 11 & $8.5(4.3-14.7)$ \\
Atypia of undetermined significance & 3 & $2.3(0.5-6.6)$ \\
Hashimato's thyroiditis & 2 & $1.6(0.2-5.5)$ \\
Otheró & 9 & $7.0(3.2-12.8)$ \\
\hline
\end{tabular}

óother includes adenomatoid goiter, lymphocytic thyroiditis, granulocytic thyroiditis, thyroid cyst, nodular goiter, follicular lesions of undetermined significance, follicular neoplasm, and papillary carcinoma.

\section{Clinical presentation of patients with thyrotoxicosis}

The most common symptom was palpitation which was present in 171 (85.5\%) of patients followed by fatigue 138 (69.0\%) and heat intolerance 118 (59.0\%) while the most common sign was anterior neck swelling 186 (93.0\%) followed by tachycardia 86 (43.0\%), exophthalmos 35 (17.5\%), and tremor 35 (17.5\%) (Table 3).

The mean duration of the anterior neck swelling was $13.4 \pm 11.1$ years. A total of $12(6.0 \%)$ patients had thyroid storm. The most common precipitating factor was infection (41.7\%) followed by drug discontinuation (8.3\%) and diabetic ketoacidosis (DKA) (8.3\%). The rest had no any identified precipitating factor.

Echocardiography was done for 89 (44.5\%) patients. Dilated cardiomyopathy (39.3\%), normal (22.5\%), Pulmonary hypertension (19.1\%), and degenerative valvular heart disease (10.1\%) were the most common findings. In addition, the most common arrythmias detected on electrocardiography (ECG) were sinus tachycardia (47.7\%) and atrial fibrillation (28.9\%) (Table 3).

Table 3: Symptoms, signs, and cardiac investigation findings of patients with thyrotoxicosis at the endocrine clinic, ACSH from December, 2017 to June, 2018, N=200. 


\begin{tabular}{lll}
\hline Clinical features & Frequency & Percentage (95\% CI) \\
\hline Symptoms & & \\
Palpitation & 171 & $\mathbf{8 5 . 5}(79.8-90.1)$ \\
Fatigue & 138 & $\mathbf{6 9 . 0}(62.1-75.3)$ \\
Heat intolerance & 118 & $\mathbf{5 9 . 0}(51.8-65.9)$ \\
Irritability & 110 & $\mathbf{5 5 . 0}(47.8-62.0)$ \\
Hyperactivity & 104 & $\mathbf{5 2 . 0}(44.8-59.1)$ \\
Sweating & 89 & $\mathbf{4 4 . 5}(37.5-51.7)$ \\
Weight loss & 70 & $\mathbf{3 5 . 0}(28.4-42.0)$ \\
Increased appetite & 66 & $\mathbf{3 3 . 0}(26.5-40.0)$ \\
Difficulty of swallowing & 10 & $\mathbf{5 . 0}(2.4-9.0)$ \\
Dyspnea & 10 & $\mathbf{5 . 0}(2.4-9.0)$ \\
Abortion & 6 & $\mathbf{3 . 0}(1.1-6.4)$ \\
Dysmenorrhea & 4 & $\mathbf{2 . 0}(0.5-5.0)$ \\
& & \\
Anterior neck swelling & 186 & $\mathbf{9 3 . 0}(88.5-96.1)$ \\
Tachycardia & 86 & $\mathbf{4 3 . 0}(36.0-50.2)$ \\
Exophthalmos & 35 & $\mathbf{1 7 . 5}(12.5-23.5)$ \\
Tremor & 35 & $\mathbf{1 7 . 5}(12.5-23.5)$ \\
Lid lag & 2 & $\mathbf{1 . 0}(0.1-3.6)$ \\
Lid retraction & 1 & $\mathbf{0 . 5}(0.0-2.8)$ \\
Echo (n=89) & & \\
Dilated cardiomyopathy & 35 & $\mathbf{3 9 . 3}(29.1-50.3)$ \\
Normal & 20 & $\mathbf{2 2 . 5}(14.3-32.6)$ \\
Pulmonary hypertension & 17 & $\mathbf{1 9 . 1}(11.5-28.8)$ \\
Degenerative valvular heart disease & 9 & $\mathbf{1 0 . 1}(4.7-18.3)$ \\
Rheumatic valvular heart disease & 6 & $\mathbf{6 . 7}(2.5-14.1)$ \\
Hypertensive heart disease & 2 & $\mathbf{2 . 2}(0.0-7.9)$ \\
ECG (n=149) & & $\mathbf{4 7 . 7}(39.4-56.0)$ \\
Sinus tachycardia & 71 & $\mathbf{2 8 . 9}(21.7-36.8)$ \\
Atrial fibrillation & 43 & $\mathbf{2 3 . 5}(16.9-31.1)$ \\
Normal & 35 & $\mathbf{0 . 7}(0.0-3.7)$ \\
Sinus bradycardia & 1 &
\end{tabular}

Note: Echo= Echocardiography, ECG= Electrocardiography

Factors Associated with Dilated Cardiomyopathy

A binary logistic regression was conducted to identify significant predictors of dilated cardiomyopathy in patients diagnosed to have thyrotoxicosis. After adjusting for confounding effect (i.e., during multivariable analysis), only atrial fibrillation and tachycardia were found to have a significant association with dilated cardiomyopathy. Accordingly, the odds of dilated cardiomyopathy was higher in patients who had atrial fibrillation $(\boldsymbol{A O R}=15.95,95 \% \boldsymbol{C I}: 5.89-38.16, \boldsymbol{p}=0.001)$ and tachycardia $(\boldsymbol{A O R}=2.73,95 \%$

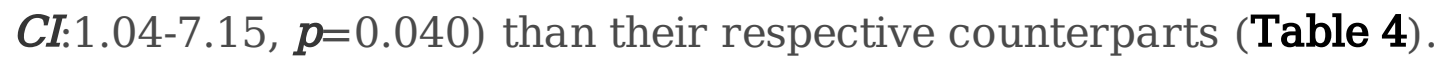


Table 4: Bivariate and multivariable Analysis to identify determinants of dilated cardiomyopathy in patients with thyrotoxicosis at the endocrine clinic, ACSH from December, 2017 to June, 2018.

\begin{tabular}{rcccc}
\hline Variables /Categories & COR (95\% CI) & $P$ value & AOR (95\% CI) & $P$ value \\
\hline $\begin{array}{r}\text { Age (in years) } \\
\leq 40\end{array}$ & 1 & 1 & & \\
$>40$ & $2.46(1.16-5.24)$ & 0.019 & $0.72(0.27-1.95)$ & 0.523
\end{tabular}

Level of T4

$\begin{array}{lllll}\leq 60 & 1 & 1 & \\ >60 & 2.99(1.39-6.41) & 0.005 & \mathbf{0 . 6 7}(0.25-1.80) & 0.436\end{array}$

Atrial fibrillation

$\begin{array}{lllll}\text { Yes } & \mathbf{2 0 . 4 2}(8.45-49.31) & 0.001 & \mathbf{1 5 . 9 5}(5.89-38.16) & 0.001 \\ \text { No } & 1 & 1 & 1\end{array}$

Tachycardia

$\begin{array}{lllll}\text { Yes } & \mathbf{5 . 4 8}(2.49-12.01) & 0.001 & \mathbf{2 . 7 3}(1.04-7.15) & 0.040 \\ \text { No } & 1 & 1 & \end{array}$

Management of patients with thyrotoxicosis and drug adherence

All patients with thyrotoxicosis received PTU as antithyroid drug and $13.0 \%$ of them experienced its side effects (like nausea, stomach upset, headache, and so on). Propranolol was the most commonly (78.5\% of the cases) used beta blocker followed by atenolol (15.0\%) and metoprolol (2.5\%). The rest didn't take any beta blocker due to normal Free T4 level and/or bradycardia. Six (3\%) of the patients underwent surgery. None of the participants received radioiodine therapy. Health information was offered to $48 \%$ of patients. Regarding TSH follow up interval, 56\% of patients had a follow up interval 
greater than 3 months. After treatment, $51.5 \%$ of the patients had their symptoms resolved within 3 months.

The adherence rate in this study was $74 \%$. Based on multivariable logistic regression analysis, lack of health information ( $\mathrm{AOR}=4.19,95 \% \mathrm{CI}$ : 1.60-10.97, $\mathrm{p}=0.009)$, being illiterate $(\mathrm{AOR}=3.47,95 \% \mathrm{CI}: 1.20-9.99, \mathrm{p}=0.026)$, TSH follow up interval $>3$ months $(\mathrm{AOR}=4.69,95 \% \mathrm{CI}: 1.59-13.37, \mathrm{p}=0.02)$, and occurrence of side effects ( $\mathrm{AOR}=11.28,95 \%$ CI: 2.97-42.85, p<0.001) were the significant reasons for non-adherence (Table 5).

Table 5: factors associated with drug non-adherence in patients with thyrotoxicosis at the Endocrine clinic of ACSH from December, 2017 to June, 2018.

\begin{tabular}{cllll}
\hline Variables /Categories & COR (95\% CI) & P value & AOR (95\% CI) & $P$ value \\
\hline Level of education & $3.41(1.16-10.01)$ & 0.025 & $3.47(1.20-9.99)$ & 0.026 \\
$\begin{array}{c}\text { Illiterate } \\
\text { Literate }\end{array}$ & 1 & & 1 & \\
$\begin{array}{c}\text { Health information } \\
\text { Given }\end{array}$ & 1 & 1 & \\
$\quad$ Not given & $3.67(1.47-9.18)$ & 0.005 & $4.19(1.60-10.97)$ & 0.009 \\
$\begin{array}{c}\text { Frequency of TSH follow up } \\
\leq 3 \text { months }\end{array}$ & 1 & & 1 & \\
>3 months & $3.83(1.38-10.62)$ & 0.001 & $4.69(1.59-13.37)$ & 0.002 \\
Side effects & & & & \\
Yes & $6.04(2.05-17.72)$ & 0.001 & $11.28(2.97-42.85)$ & $<0.001$ \\
No & 1 & & 1 & \\
\hline
\end{tabular}

\section{Discussion}

The main purpose of this study was to assess the pattern, clinical presentation, management, complication, and drug adherence of patients with thyrotoxicosis. It was also concerned with identification of factors associated with dilated cardiomyopathy and drug non-adherence.

In this study, the majority (89\%) of patients were females. Mean age at presentation was 45 years and those who were above 40 years accounted for $58.5 \%$ of the cases. This is similar to a study done in Addis Ababa, in which $90 \%$ of patients were females and patients above 40 year of age accounted for $59 \%$ of the cases with mean age of 43.1 years (1). Another study in Ghana also showed similar results where the mean age was 41.5 and most of the patients (87.8\%) were females (17).

The most common symptom of patients with thyrotoxicosis was palpitation which was present in $85.5 \%$ of the patients followed by fatigue (69.0\%) and heat intolerance (59.0\%). Similarly, Addis Ababa's study revealed palpitation and heat intolerance as the most common symptoms of patients with thyrotoxicosis 
(1). As well, a study done in South-Western Nigeria found palpitation and fatigue as the most common symptoms which occurred in $85.0 \%$ and $63.4 \%$ of patients respectively (18). A study done in France on 1240 patients also supported the above findings and reported palpitation followed by weakness as the most common clinical presentation of thyrotoxicosis (4).

The most common sign in our study was palpable thyroid (93.0\%) followed by tachycardia (43.0\%), exophthalmos (17.5\%), and tremor (17.5\%). According to Addis Ababa's study, palpable thyroid (goiter) was also the commonest sign of thyrotoxicosis which was detected in $99 \%$ of the cases. Congruently, the most common sign in Nigerian study was palpable thyroid and found in $97 \%$ of the cases (18). In contrary, a study done at Maharashtra, India found tremor as the most common sign of thyrotoxicosis, in about $63.0 \%$ of patients (19).

The most common arrythmia detected on electrocardiography (ECG) was sinus tachycardia followed by atrial fibrillation and this finding goes parallel with several case studies on thyrotoxic cardiomyopathy $(20-22)$. This prevalence of atrial fibrillation on ECG of patients with thyrotoxicosis is also comparable to a study done in India where it was $28 \%$ (23). The prevalence of dilated cardiomyopathy in our study (which is around $40 \%$ ) is higher compared to other studies where it is reported to be $1 \%$ (24). Even taking the whole participants as denominator, still the prevalence is higher which is about $17.5 \%$. The main reasons for this higher prevalence are two. The first reason is late presentation with mean duration of goiter (i.e., anterior neck swelling) about 13 years. This explanation is supported by a study done in England that found older age, male sex, and longstanding thyrotoxicosis as factors that increase the risk of dilated cardiomyopathy (24). The second reason is that echocardiography was done only for patients with signs and symptoms of thyrocardiac diseases. In this study, the factors significantly associated with dilated cardiomyopathy were atrial fibrillation and tachycardia. Thyroid storm was present in $6 \%$ of this study participants. This figure is comparable to a national study done in Japan where it was $5.4 \%(10)$.

In our study, PTU was the only thionamide used to treat thyrotoxicosis. This is similar to the study done in Addis Ababa, Ethiopia where almost all of the patients received PTU (1). This finding contradicts to a survey done by members of the Endocrine Society (ES), American Thyroid Association (ATA) and American Association of Clinical Endocrinologists (AACE 6) where the most commonly used drug was methimazole (83.5\% of the cases) while the use of PTU was limited to $2.7 \%(25)$. This changing in trend towards methimazole use in the developed world is because methimazole is associated with high rate of free T4 normalization and less side effects (26)(27). But in developing countries like Ethiopia, methimazole is hardly available and almost all thyrotoxicosis cases are treated with PTU (28). Currently, the recommendation is to use methimazole as the first line anti-thyroid with an exception during pregnancy in which PTU is preferred because of rare reports of birth defects associated with methimazole (29). Additionally, in life threatening conditions like thyroid storm, PTU is also preferred since it inhibits conversion of T4 to T3 (30).

The interval of TSH follow up was $>3$ months in $112(56 \%)$ of the patients and it was against the recommendations by the American Association of Clinical Endocrinologists and the American Thyroid 
Association which recommend TSH determination every 4 to 6 weeks for all patients till normalization of TSH and T4 $(25,31)$. The most common reasons not to do it based on the recommendation were limited availability of the investigation and financial constraints (more than half of the patients had a lower monthly income which is below 1000 ETB).

Finally, the non-adherence rate of PTU in this study was $26 \%$. The identified reasons for non-adherence of PTU were being illiterate, lack of health information, side effects, and infrequent TSH follow up. This finding somehow strengthens the claim that PTU is not a good choice of drug in controlling severe form of the disease, has poor adherence rate, and several adverse effects (32).

\section{Strengths of the study}

It is the first study to assess the pattern, clinical presentation and management of thyrotoxicosis in Tigray and can serve as baseline for future studies related to the current topic. It has also tried to assess many aspects of thyrotoxicosis by collecting primary data using a pre-tested and comprehensive questionnaire.

\section{Limitations of the study}

Echocardiography, electrocardiography, and cytology were not done for all patients. As a result, this study may not show the true prevalence of cytologic types and cardiac complications in patients with thyrotoxicosis. Due to infrequent TSH follow-up, this study didn't address time to normalization of free T4 and TSH.

\section{Conclusion}

This study has shown that most of the patients with thyrotoxicosis were females. The most common symptom and sign of thyrotoxicosis were palpitation and goiter respectively. All patients took PTU as anti-thyroid drug and one-fourth of them were non-adherent to this drug due to illiteracy, lack of health information, side effects, and longer follow up interval. Dilated cardiomyopathy was the commonest abnormal echocardiography finding in patients with thyrotoxicosis and it was significantly associated with atrial fibrillation and tachycardia. The prevalence of thyrocardiac disease was higher even taking all participants as denominator which may be attributed to the longstanding thyrotoxicosis. Overall, the findings of this study complement and strengthen studies done in another part of Ethiopia and other African countries.

\section{Recommendations}

Patients should be given adequate health information about thyrotoxicosis and anti-thyroid drugs including their side effects. Hospitals and other concerned bodies should also avail TSH test and methimazole at an affordable cost. 


\section{List Of Abbreviations}

ACSH Ayder Comprehensive Specialized Hospital

AUS Atypia of undetermined significance

DKA Diabetic Ketoacidosis

ECG Electrocardiography

ETB Ethiopian Birr

FNAC Fine Needle Aspiration Cytology

MMAS-8 Morisky Medication Adherence Scale of 8-item

PTU Propylthiouracil

SPSS Statistical Package for Social Sciences

SSA sub-Saharan Africa

T4 Thyroxine

TSH Thyroid Stimulating Hormone

\section{Declarations}

Ethics approval and consent to participate

Ethical clearance was obtained from Ethical Review Committee of Mekelle University, College of health sciences with ethical clearance number ERC: $1408 / 2018$. The purpose of the study was explained to participants. Informed consent was obtained from participants. Participant involvement in the study was also on voluntary basis. Confidentiality of information was assured. All methods are done in accordance with relevant guidelines and regulations.

\section{Consent for publication}

Not applicable.

\section{Availability of data and materials}

The datasets used and/or analysed during this study are available from the corresponding author on reasonable request.

Competing interests 
The authors declare that they have no competing interests.

\section{Funding}

Not applicable.

\section{Authors' contributions}

BTA selected the topic, planned the study protocol, oversaw the study, entered the data, and reviewed the literatures. BTA, MME, and HT conducted the analysis and interpreted the results. BTA, MME, and MAA drafted the final manuscript. All authors reviewed the manuscript.

\section{Acknowledgements}

We would like to forward our gratitude to Sr. Askual, Dr Kelali G/haweriat and Dr Ajawin Anthoy for their sincere help during data collection. We would also like to thank Mekelle University, College of Health Sciences, Internal Medicine department members for their sincere help in selecting this topic and reviewing relevant literatures.

\section{Authors' information}

Bisrat Tesfay Abera, bis9live@gmail.com, Mekelle University, College of Health Sciences, Mekelle, Ethiopia.

Hirut Teame Gebru, hirutteame@gmail.com, Adigrat University, College of Health Sciences, Adigrat, Ethiopia.

Merhawit Atsbha Abera, merhawitats@gmail.com, Mekelle University, College of Health Sciences, Mekelle, Ethiopia.

Gebretsadik Berhe, gebretsadik_b@yahoo.com, Mekelle University, College of Health Sciences, Mekelle, Ethiopia.

Girmatsion Fisseha Abreha, girmaf4@yahoo.com, Mekelle University, College of Health Sciences, Mekelle, Ethiopia.

Mohamedawel Mohamedniguss Ebrahim, mohamedawel@gmail.com, Mekelle University, College of Health Sciences, Mekelle, Ethiopia.

Hiluf Ebuy Abraha, hilebuy@gmail.com, Mekelle University, College of Health Sciences, Mekelle, Ethiopia.

\section{References}

1. Kebede D, Abay Z, Feleke Y. Pattern, clinical presentations and management of thyroid diseases in national endocrine referral clinics, Tikur Anbessa Specialized Hospital, Addis Ababa, Ethiopia. 
Ethiopian medical journal. 2012 Oct 1;50(4):287-95.

2. Kasper, fauci, hauser L. Harrison Princiles of Internal Medicine. 19th ed. McGraw-Hill Education; 2015. 2283-2308 p.

3. Vanderpump MPJ. The epidemiology of thyroid disease. Br Med Bull. 2011;99(1):39-51.

4. Goichot B, Caron P, Landron F, Bouée S. Clinical presentation of thyrotoxicosis in a large representative sample of outpatients in France: Relationships with age, aetiology and hormonal parameters. Clin Endocrinol (Oxf). 2016;84(3):445-51.

5. Ogbera A, Isiba A. The scope of cardiac complications of thyrotoxicosis in Lagos, Nigeria. InSociety for Endocrinology BES 2007 Mar 1 (Vol. 13). BioScientifica.

6. Frost L, Vestergaard P, Mosekilde L. Thyrotoxicosis and risk of atrial fibrillation or flutter: a population-based study. Archives of internal medicine. 2004 Aug 9;164(15):1675-8.

7. Kandan V, Sathyamurthy P, Rajkumar M, Narayanan L. Cardiovascular manifestations in thyrotoxicosis. 2016;4(7):3032-8.

8. Goland S, Shimoni S, Kraco O V, Goland S. Dilated cardiomyopathy in thyrotoxicosis Non-surgical CHOP cures right ventricular outflow obstruction. 1999;444-6.

9. Carroll R, Matfin G. Review: Endocrine and metabolic emergencies: Thyroid storm. Ther Adv Endocrinol Metab. 2010;1(3):139-45.

10. Chiha M, Samarasinghe S, Kabaker AS. Thyroid Storm: An Updated Review. J Intensive Care Med. 2015;30(3):131-40.

11. Pearce EN. Diagnosis and management of thyrotoxicosis Clinical review Diagnosis and management of thyrotoxicosis. 2006;5128(April 2008):1-11.

12. Jonklaas J, Bianco AC, Bauer AJ, Burman KD, Cappola AR, Celi FS, et al. Guidelines for the Treatment of Hypothyroidism: Prepared by the American Thyroid Association Task Force on Thyroid Hormone Replacement. Thyroid. 2014;24(12):1670-751.

13. Premawardhana LD, Lazarus JH. Management of thyroid disorders. Postgraduate medical journal. 2006 Sep 1;82(971):552-8.

14. Reine B La, Affairs M, Serono M, Diseases M. Survey of Clinical Practice Patterns in the Management of 992 Hyperthyroid Patients in France. Endocr Pract. 2017;17(3):152-9.

15. Allan S. Brett M. Methimazole vs. Propylthiouracil for Thyrotoxicosis. NEJM Endocr Watch. 2007;92:2157-62.

16. Ogbera AO, Kuku SF. Epidemiology of thyroid diseases in Africa. Indian journal of endocrinology and metabolism. 2011 Jul;15(Suppl2):S82.

17. Gyasi RK. Thyroid Disorders in Accra, Ghana: A Retrospective Histopathological Study at the KorleBu Teaching Hospital. J Med Biomed Sci. 2013;2(1):1-7.

18. Ogbera A01, Fasanmade O AO. Pattern of thyroid disorders in the SouthWestern region of Nigeria. NCBI. 2007;17(2):327-30. 
19. Dhadke SV, Dhadke VN, Korade MB, Sangle SA. Research Article Clinical Profile of Thyroid Disorders. Int J Curr Res. 2014;6(09):8484-8.

20. Faizel O, Michael DG, Michael CS, et al. Cardiac dysrhythmias and thyroid dysfunctionThe hidden menace? J Clin Endocrinol Metab 2002;87:963-7.

21. OF F, DE J. Thyrotoxic cardiomyopathy - A case report. 2016;6(3):49-51.

22. Ademolu A. Thyrotoxic Cardiomyopathy: A Study of Three Cases. Gerontol Geriatr Stud. 2017;1(3):46-9.

23. Kandan V, Sathyamurthy P, Rajkumar M, Narayanan L. Cardiovascular manifestations in thyrotoxicosis. 2016;4(7):3032-8.

24. Bes E. Endocrine Abstracts. Endocr Abstr [Internet]. 2014;34(March):408. Available from: https://www.endocrine-abstracts.org/ea/0034/SFEBES2014AbstractBook.pdf

25. Bahn RS, Burch HB, Cooper DS, Garber JR, Greenlee MC, Klein I, Laurberg P, McDougall IR, Montori VM, Rivkees SA. Thyrotoxicosis and other causes of thyrotoxicosis: management guidelines of the American Thyroid Association and American Association of Clin.

26. Allan S. Brett M. Methimazole vs. Propylthiouracil for Thyrotoxicosis. NEJM Endocr Watch. 2007;92:2157-62.

27. Emiliano AB, Governale L, Parks M, Cooper DS. Shifts in Propylthiouracil and Methimazole Prescribing Practices: Antithyroid Drug Use in the United States from 1991 to 2008. 2014;95(May 2010):2227-33.

28. Gebreyohannes EA, Ayele EM, Tesfaye SA. Normalization of thyroid function tests among thyrotoxicosis patients attending a University Hospital in North-West Ethiopia. 2019;1-8.

29. Abalovich M, Amino N, Barbour LA, Cobin RH, De Groot LJ, Glinoer D, Mandel SJ, Stagnaro-Green A Management of thyroid dysfunction during pregnancy and postpartum: an Endocrine Society Clinical Practice Guideline. J Clin Endocrinol Metab. 2007; 92(8 Suppl).

30. Cooper DS, Rivkees SA. Putting Propylthiouracil in Perspective. 2009;94(June):1881-2.

31. Garber JR, Cobin RH, Gharib H, Hennessey JV, Klein I, Mechanick JI, Pessah-Pollack R, Singer PA, Woeber for the American Association of Clinical Endocrinologists and American Thyroid Association Taskforce on Hypothyroidism in Adults KA. Clinical practice .

32. Cooper DS. Antithyroid drugs. N Engl J Med. 2005; 352:905-917. 